Rev. Elev. Méd. vét. Pays trop., 1971, 24 (3) : 467.76

\title{
Possibilités d'accroissement de la productivité économique du Zébu Malgache par amélioration génétique et alimentation intensive
}

\author{
par H. SERRES $(*)$, J. GILIBERT $(*)$, G. CHATILLON $(*)$
}

\begin{abstract}
RESUME
Des animaux métis Zébu Brahman $\times$ Zébu Malgache de 16 mois environ et des métis Zébu Malgache $\times$ Frison (ou Normand) de 6 à 7 mois énviron ont été nourris intensivement. Les résultats des croissances. des indices de consommation. et les caractéristiques de carcasses sont donnés. Comparativement à des Zćbus Malgaches de même âge, nourris de façon comparable, des gains importants sont obtenus sur tous les plans. Le croisement Brahman, réatisable en élevage extensif, paraît très recommandé.
\end{abstract}

Nous avons eu l'occasion d'étudier les modalités d'engraissement du Zébu Malgache soumis à une alimntation intensive (1). Comme il fallait s'y attendre les résultats sont meilleurs avec des animaux jeunes, mais ils demandent une embouche prolongée pour obtenir des carcasses d'un poids suffisant. Les animaux plus âgés permettent d'obtenir le poids requis en un temps plus court, mais avec par contre un indice de consommation élevé. Dans tous les cas, croîts quotidiens moyens et indices de consommation sont nettement moins avantageux que ceux obtenus avec du bétail taurin.

Or, il se trouve qu'à Madagascar, le Zébu Malgache a été croisé, sur une échelle déjà non négligeable, avec le Brahman du Texas pour les régions côtières, avec le Normand et le Frison sur les Hauts Plateaux. On a pu montrer (2) que le croisement Zébu $X$ Malgache $X$ Brahman permettait d'obtenir, en élevage extensif, un poids d'environ 25 p. 100 supérieur à âge égal et, finalement, une taille et un poids adultes très nettement plus élevés. Le gain obtenu par croisement avec des taurins

(*) I.E.M.V.T., Région de recherches de Madagascar. laitiers, sur les plateaux, bien qu'important, n'a jamais été chiffré avec précision.

Dans ce qui suit nous essaierons de montrer cornment ces croisements peuvent influencer les capacités de ce bétail à tirer profit d'une alimentation intensive; le gain le plus intéressant à espérer nous paraissant la précocité économique, nous nous sommes adressés à de jeunes animaux âgés de 16 mois en début d'essai.

\section{AMELIORATION \\ PAR CROISEMENT BRAHMAN}

Nous avons choisi 10 animaux métis Zébu Brahman $\times$ Zébu Malgache, dont 8 avaient $5 / 8$ de sang Brahman et les deux autres $3 / 4$. Ils provenaient de lélevage extensif pratiqué sur les pâturages naturels du Moyen Ouest Malgache. Ces animaux castrés à Kianjasoa, ont été maintenus en stalle de $10 \times 10 \mathrm{~m}$.

Leur alimentation comprenait un concentré à 80 p. 100 de maïs, 16 p. 100 de tourteau d'arachide, 4 p. 100 de minéraux, déjà décrit (1), le tout ad libitum, et $8 \mathrm{~kg}$ de Tripsacum Iaxum par jour.

Ce bétail se révéla, dès sa mise en stabulation, particulièrement calme et s'adapta très vite à l'alimentation intensive. 
Les résultats de cette embouche sont retracés au tableau I. On y remarque des gains de poids quotidiens qui se maintiennent longtemps audessus de $1000 \mathrm{~g}$ par jour, pour ne fléchir que légèrement après 3 mois, et s'établir à une moyenne de $990 \mathrm{~g} /$ jour pour la période de cinq mois et demi, avec un indice de consommation global de 8,7 .

On peut rapprocher ces résultats de ceux obtenus avec un lot de zébus jeunes ( 2 ans et demi) engraissé également à Kianjasoa, avec le même type d'alimentation mais rationnée.

TABLEAU $N^{\circ}$ I

\begin{tabular}{|l|c|c|c|c|c|c|c|}
\hline \multicolumn{1}{|c|}{$\mathrm{N}^{\circ}$ pesées } & & 1 & 2 & 3 & 4 & 5 & 6 \\
\hline $\begin{array}{l}\text { C.Q.M. } \\
\text { cumulatifs } \\
\text { (g) }\end{array}$ & Zëbu malgache & 635 & 695 & 750 & 642 & 643 & 546 \\
\cline { 2 - 8 } & Métis Brahman & 1.075 & 1.067 & 1.065 & 935 & 910 & 888 \\
\hline $\begin{array}{l}\text { Indice de } \\
\text { consommation }\end{array}$ & Zébu Malgache & 6,8 & 7,5 & 7,1 & 9 & 9,2 & 11 \\
\cline { 2 - 8 } & Métis Brahman & 7,2 & 7,9 & 7,8 & 8,4 & 8,5 & 8,7 \\
\hline
\end{tabular}

C.Q.M. = croît quotidien moyen.

L'alimentation ad libitum favorise de façon certaine les métis sur le plan des croîts quotidiens. Mais la différence est tellement importante qu'elle ne peut pas s'expliquer par cela seulement, puisque le rationnement, largement calculé, n'avait pour but que d'éviter un inutile gaspillage.

En ce qui concerne les indices de consommation, les animaux soumis au rationnement se trouvent par contre avantagés. Or, on constate que si au début les Zébus Malgaches se sont maintenus à un niveau d'indice effectivement un peu inférieur, après trois mois cela s'est inversé.

Les métis Brahman n'ont accru que très peu leur indice, tandis que celui des zébus malgaches s'élevait rapidement pour s'établir à 11 au bout de 5 mois et demi. Cela traduit qu'après 3 mois d'engraissement le jeune Zébu Malgache commence à faire beaucoup de graisse et moins de muscle, alors que le métis Brahman continue à faire du muscle en n'engraissant que lentement.

Les carcasses des métis Brahman ont donné les résultats notés au tableau $\mathrm{n}^{\circ} \mathrm{II}$.

Ces résultats sont excellents, ce que confirmait l'examen visuel.

On concluera donc que l'utilisation des métis Brahman est souhaitable pour un Centre d'embouche car:
TABLEAU $N^{\circ}$ II

\begin{tabular}{|ll|c|}
\hline Poids vif au départ & $(\mathrm{kg})$ & 398 \\
Poids vif vide & $(\mathrm{kg})$ & 349,6 \\
Contenus digestifs & $(\mathrm{kg})$ & 48,4 \\
\multicolumn{1}{|c|}{ p.100 P.V. départ } & & 12,1 \\
Poids carcasse chaude & $(\mathrm{kg})$ & 233,7 \\
Poids carcasse froide & $(\mathrm{kg})$ & 229,1 \\
Rendement commercial & $(\mathrm{p} .100)$ & 57,5 \\
Rendement à jeun & $(\mathrm{p} .100)$ & 61,5 \\
Rendement vrai & $(\mathrm{p} .100)$ & 65,5 \\
Longueur de la carcasse & $(\mathrm{cm})$ & 116,8 \\
Indice d'état de viande & 1,96 \\
Epaisseur de la cuisse & $(\mathrm{cm})$ & 23,3 \\
Indice de muscle & $(\mathrm{p} .100)$ & 20 \\
Gras de rognons & $(\mathrm{kg})$ & 6,37 \\
Indice de gras & & 2,8 \\
\hline
\end{tabular}

- ils peuvent être produits en élevage extensif (peu onéreux);

- ils ont des croîts quatidiens élevés;

- les indices de consommation se maintiennent longtemps à un niveau convenable;

- ils fournissent des carcasses lourdes, riches en muscles et grasses sans excès. 


\section{LES ANIMAUX}

Six veaux mâles ont été achetés chez les paysans de la région de Tananarive. Ils avaient environ six mois et leur poids variait de 52 à $88 \mathrm{~kg}$. Tous étaient en très mauvais état, avec un poil hérissé, un abdomen volumineux, une amyotrophie nette. Deux étaient des métis Normands, les quatre autres pie-noir provenaient de croisement Frison. Ils possédaient $3 / 4$ de sang taurin selon les dires des propriétaires, mais aucune certitude ne peut en être donnée. A ce sujet l'aspect extérieur était cependant en accord avec ces déclarations.

Ces animaux ont été nourris intensivement pendant 294 jours.

Trois autres animaux ont complété le lot quelques jours plus tard. Leurs origines étaient connues: il s'agissait de demi-sang FrisonZébu. Ils paraissaient avoir moins souffert et à sept mois pesaient de 95 à $113 \mathrm{~kg}$. Ils ont dans la suite les $n^{\text {os }} 7,8,9$. Ils ont été nourris intensivement pendant 252 jours.

Aucun des 9 veaux n'a été castré.

\section{PLAN DE RATIONNEMENT}

\section{a) Avant $100 \mathrm{~kg}$}

On a retenu :

Mélange concentré :

— Tourteau d'arachides . . . . . 27

- Farine basse de riz . . . . . 68

- Coquilles marines . . . . . 4,5

- Sel . . . . . . . . . . . 0,5

Ce qui représente:

$$
\begin{array}{rll}
\mathrm{MS} / \mathrm{kg} & : & 0.9 \\
\mathrm{UF} / \mathrm{kg} & : & 0,99 \\
\mathrm{MAD} / \mathrm{kg} & : & 170 \mathrm{~g}
\end{array}
$$

La ration retenue est la suivante:

TABLEAU $N^{\circ}$ III

\begin{tabular}{|l|c|c|c|c|}
\hline \multirow{2}{*}{ Mélange } & $\mathrm{kB}$ & MS & UF & MAD \\
\hline Tripsacum & 1,6 & 1,44 & 1,58 & 272 \\
\hline & 3,0 & 0,66 & 0,45 & 18 \\
\hline & & 2,10 & 2,03 & 290 \\
\hline
\end{tabular}

\section{AMELIORATION \\ PAR CROISEMENT FRISON (OU NORMAND)}

II existerait actuellement sur la bordure orientale des Hauts Plateaux de Madagascar, de 30.000 à 40.000 vaches métisses, issues de croisements variés avec des races européennes. La politique d'amélioration de ce cheptel laitier a conduit depuis une dizaine d'années au développement d'un centre d'insémination artificielle utilisant taureaux Normands et Frisons.

Les veaux mâles issus de ce cheptel laitier sont élevés généralement dans des conditions très précaires jusqu'à l'âge de 4 à 10 mois, atteignant alors des poids de 50 à $120 \mathrm{~kg}$. Le veau est laissé pendant la journée avec sa mère qu'il peut têter. Il en est séparé la nuit. La vache est traite à la main le matin et le lait obtenu est vendu. Comme elle est mal nourrie, sa productivité en lait est faible et le veau soumis à la portion congrue. La sousalimentation est une des causes principales des fortes mortalités enregistrées. Ces animaux sont généralement commercialisés pour la boucherie, le plus souvent dans un état misérable.

Chaque année, il y aurait quelques 6.000 veaux mâles dont la valorisation par le paysan est faible et dont la contribution au marché de la viande est négligeable; mais ce sont les seuls veaux commercialisés à Tananarive.

On peut penser que ces veaux souvent issus de l'insémination artificielle et produits par des vaches possédant déjà des caractères améliorés, devraient avoir une précocité très supérieure à celle du Zébu Malgache, et par là, un réel potentiel de croissance qui ne demanderait, pour se révéler, qu'une alimentation rationnelle.

Par ailleurs, les surfaces herbagères des Hauts Plateaux sont toujours faibles, et de valeur très inégale, en particulier selon la saison. L'intensification rizicole ne laisse pas espérer qu'elles puissent prendre de l'extension dans un proche avenir.

Dans ce contexte, il fallait rechercher dans quelle mesure ce potentiel de croissance pouvait être exploité à partir des sous-produits disponibles (sons de riz, tounteaux, paille de riz) at des fourrages à haute productivité. 


\section{b) Autres tranches de poids}

- On retient un apport de tourteau de $520 \mathrm{~g} /$ animal/jour, niveau qui permet de couvrir approximativement les besoins MAD.

- On retient également en issues de riz, le mélange farine basse $2 / 3$ pour $1 / 3$ de son, dont les caractéristiques doivent être les suivantes :

$\mathrm{MS} / \mathrm{kg}: \quad 0,9$

$\mathrm{UF} / \mathrm{kg}: \quad 0,79$

$\mathrm{MAD} / \mathrm{kg}: 66 \mathrm{~g} \quad$ (MAT : $100 \mathrm{~g}$ )
$\mathrm{MG} / \mathrm{kg}: 109 \mathrm{~g}$

On lui incorpore le même mélange minéral que ci-dessus.

\section{Tripsacum}

Il entre de façon constante pour environ: 28 p. 100 de la matière sèche de la ration (26 à 30 et 22 p. 100 des UF) (20-25).

On a espéré un gain de $900 \mathrm{~g}$ par jour.

Les rations retenues sont alors les suivantes:

TABLEAU N ${ }^{\circ}$ IV

\begin{tabular}{|l|c|c|c|c|c|c|c|c|c|c|c|c|}
\hline & \multicolumn{4}{|c|}{$100 \mathrm{~kg}$} & \multicolumn{4}{c|}{$150 \mathrm{~kg}$} & \multicolumn{5}{c|}{$200 \mathrm{~kg}$} \\
\hline \multirow{2}{*}{$\begin{array}{l}\text { Tourteau } \\
\text { d'arachide }\end{array}$} & $\mathrm{Kg}$ & MS & UF & MND & $\mathrm{Kg}$ & MS & UF & MAD & $\mathrm{Kg}$ & MS & UF & MAD \\
\hline $\begin{array}{l}\text { Melange } \\
\text { d'issues } \\
\text { de riz }\end{array}$ & 0,52 & 0,47 & 0,55 & 210 & 0,52 & 0,47 & 0,55 & 210 & 0,52 & 0,47 & 0,55 & 210 \\
\hline \begin{tabular}{l} 
Tripsacum \\
\hline
\end{tabular} & 1,7 & 1,53 & 1,35 & 114 & 2,7 & 2,43 & 2,14 & 178 & 3,6 & 3,24 & 2,84 & 238 \\
\hline
\end{tabular}

TABLEAU N ${ }^{\circ} \mathrm{V}$

\begin{tabular}{|l|c|c|c|c|c|c|c|c|c|c|c|c|}
\hline & \multicolumn{6}{|c|}{$250 \mathrm{~kg}$} & \multicolumn{3}{|c|}{$300 \mathrm{~kg}$} & \multicolumn{3}{|c|}{$350 \mathrm{~kg}$} \\
\hline $\begin{array}{l}\text { Tourteau } \\
\text { d'arachide }\end{array}$ & $\mathrm{kg}$ & MS & UF & MAD & $\mathrm{kg}$ & MS & UF & MAD & $\mathrm{kg}$ & MS & UF & MAD \\
\hline $\begin{array}{l}\text { Mélange } \\
\text { d'issues } \\
\text { de riz }\end{array}$ & 0,52 & 0,47 & 0,55 & 210 & 0,52 & 0,47 & 0,55 & 210 & 0,52 & 0,47 & 0,55 & 210 \\
\hline \begin{tabular}{l} 
Tripsacum \\
\hline
\end{tabular} & 84,5 & 4,05 & 3,55 & 297 & 5 & 4,50 & 3,95 & 330 & 5,2 & 4,68 & 4,10 & 343 \\
\hline
\end{tabular}

\section{CONDUITE DES ANIMAUX}

Après une période préliminaire d'adaptation de 10 jours, l'expérience proprement dite démarre.

Les animaux sont tenus en loges individuelles avec de l'eau à volonté et une litière de copeaux de bois. Ils sont pesés tous les 14 jours, à la même heure, les rations quotidiennes distribuées en une seule fois. Les refus sont contrôlés, mais sont toujours quasi nuls. Une période d'exercice d'une heure par jour est prévue, pour les animaux en commun, le matin, dans un parc ensoleillé.

\section{RESULTATS}

Tous les animaux ont eu une croissance extrêmement régulière avec des gains quotidiens très constants, dépassant quelque peu $1.000 \mathrm{~g}$ par jour pour certains, se situant entre 900 et $1.000 \mathrm{~g}$ pour les autres.

Nous indiquerons ci-après les performances individuelles. 
TABLEAU $N^{\circ} V I$

\begin{tabular}{|c|c|c|c|c|c|}
\hline $\begin{array}{l}N^{a} d u \\
\text { taurillon }\end{array}$ & $\begin{array}{l}\text { Poids } \\
\text { d'achat } \\
\text { (kg) }\end{array}$ & $\begin{array}{l}\text { Poids } \\
\text { final } \\
(\mathrm{kg})\end{array}$ & $\begin{array}{l}\text { Gain } \\
(\mathrm{kg})\end{array}$ & $\begin{array}{c}\text { Jours } \\
\text { d'engraissement } \\
\text { ( } n \text { ) }\end{array}$ & $\begin{array}{l}\text { C.Q.M. } \\
\text { (g) }\end{array}$ \\
\hline 1 & 81 & 361 & 280 & 294 & 950 \\
\hline 2 & 80 & 394 & 314 & " & 1.070 \\
\hline 3 & 74 & 355 & 281 & $"$ & 960 \\
\hline 4 & 52 & 319 & 267 & $"$ & 910 \\
\hline 5 & B8 & 363 & 275 & $"$ & 940 \\
\hline 6 & 67 & 370 & 303 & $"$ & 1.030 \\
\hline 7 & 113 & 362 & 249 & 252 & 990 \\
\hline 8 & 95 & 320 & 225 & $"$ & 890 \\
\hline 9 & 100 & 345 & 245 & $"$ & 970 \\
\hline
\end{tabular}

C.Q.M. = croit quotidien moyen.

En moyenne les $1 / 2$ sang ont eu des croîts quotidiens de $950 \mathrm{~g}$ alors que les animaux ayant vraisemblablement un degré de sang un peu plus élevé ont atteint $975 \mathrm{~g}$. La différence est donc très faible.

L'évolution des croissances et des consommations pendant l'essai, entre les poids moyens de $113,8 \mathrm{~kg}$ à $362,5 \mathrm{~kg}$ sont retracés au tableau VII (p. 474).

On voit que les croîts quotidiens ont augmenté pendant deux mois, puis ils ont amorcé une décroissance très lente. Les indices de consommation sont au début extrêmement bas. Les mesures ont été cependant faites avec le plus grand soin. Il faut attribuer cela à plusieurs facteurs concourants: d'abord le fait que les veaux étaient au départ en mauvais état, ils ont dû se réhydrater et effectuer une croissance compensatrice; ensuite ils ont eu un comportement digestif de type monogastrique.

Les indices de consommation se sont peu à peu relevés, pour atteindre 8,5 en fin d'essai, après 8 mois. Mais l'indice général reste inférieur à 5 , ce qui est vraiment excellent.

On peut effectuer un rapprochement entre ces animaux, et un lot de taurillons zébu malgache, élevés de 10 à 19 mois, nourris avec une ration à base d'issues de riz et de tourteau d'arachide et $5 \mathrm{~kg}$ de verdure par jour, donc tout à fait comparable en qualité et en quantité.

On peut comparer les croîts quotidiens moyens cumulatifs à partir du poids moyen de $145 \mathrm{~kg}$ et de lâge approximatif de 10 mois (tableau VIII) :

TABLEAU $\mathrm{Y}^{0}$ VIII

\begin{tabular}{|c|c|c|c|c|c|c|c|c|c|}
\hline \multirow{3}{*}{$\begin{array}{c}\text { C.Q.M. } \\
(\mathrm{g})\end{array}$} & $\mathrm{N}^{\circ}$ pesées & 1 & 2 & 3 & 4 & 5 & 6 & 7 & 8 \\
\hline & $\begin{array}{l}\text { Zêbu } \\
\text { malgache }\end{array}$ & 1142 & 985 & 982 & 919 & 857 & 797 & 734 & 705 \\
\hline & Mêtis & 973 & 984,5 & 1013,6 & 1018,7 & 999,6 & 987,5 & 965,1 & 932,5 \\
\hline
\end{tabular}

C.Q.M. = croît quotidien moyen.

Les Zébus Malgaches maintiennent une croissance élevée pendant 4 mois, au-dessus de $900 \mathrm{~g}$ par jour, mais ensuite les croîts quotidiens diminuent assez vite, pour se situer à un niveau final de $700 \mathrm{~g}$. Les métis se main- tiennent nettement au-dessus de $900 \mathrm{~g}$ jusqu'au bout.

Dans cet exemple encore, le croisement fait apparaître une amélioration importante des performances par rapport au Zébu Malgache pur. 
TABLEAU $\mathrm{N}^{\mathrm{a}} \mathrm{VII}$

\begin{tabular}{|c|c|c|c|c|c|c|c|c|c|c|c|c|c|c|c|c|c|c|c|}
\hline Dates & 1.8 & & 29.8 & & 26.9 & & 24.10 & & 21.11 & & 19.12 & & 16.1 & & 13.2 & & 13.3 & & 10.4 \\
\hline Puids du lot $(\mathrm{kg})$ & 1025 & & 1315 & & 1569 & & 1829 & & 2109 & & 2379 & & 2620 & & 2862 & & 3079 & & 3263 \\
\hline $\begin{array}{l}\text { Gains par } \\
\text { përiode (kg) }\end{array}$ & & 290 & & 254 & & 260 & & 280 & & 270 & & 241 & & 242 & & 217 & & 184 & \\
\hline C.Q.M. (g) & & 1111 & & 973 & & 996 & & 1072 & & 1034 & & 923 & & 927 & & 831 & & 704 & \\
\hline U.F. consommées & & 650 & & 874 & & 895 & & 1120 & & 1185 & & 1379 & & 1420 & & 1526 & & 1572 & \\
\hline I.C./përiode & & 2,24 & & 3,44 & & 3,44 & & 4,0 & & 4,38 & & 5,7 & & 5,86 & & 7,0 & & 8,5 & \\
\hline
\end{tabular}

Croŝt quotıdien moyen dt1 1.8 .68 au $10.4 .69: 986,7 \mathrm{~g}$

Indice de consumation moyen 4,74 . 
Les taurillons métis ont été abattus lorsqu'on a eu la certitude d'obtenir des carcasses d'un poids supérieur à $200 \mathrm{~kg}$.

Les résultats sont les suivants :

TABLEAU $N^{*} I X$

\begin{tabular}{|ll|c|}
\hline Poids vif au départ & $(\mathrm{kg})$ & 390,2 \\
Poids vif vide & $(\mathrm{kg})$ & 347 \\
Contenus digestifs & $(\mathrm{kg})$ & 43,2 \\
p.100 P.V. dëpart & & 11 \\
Poids carcasse chaude & $(\mathrm{kg})$ & 219,3 \\
Poids carcasse froide & $(\mathrm{kg})$ & 217,6 \\
Rendement commercial & $(\mathrm{p} .100)$ & 55,7 \\
Rendement à jeun & $(\mathrm{p} .100)$ & 58,6 \\
Rendement vrai & $(\mathrm{p} .100)$ & 62,7 \\
Longueur de la carcasse & $(\mathrm{cm})$ & 117,4 \\
Indice d'état de viande & & 1,85 \\
Epaisseur de la cuisse & $(\mathrm{cm})$ & 22,8 \\
Indice de muscle & $(\mathrm{p} .100)$ & 19,4 \\
Gras de rognons & $(\mathrm{kg})$ & 4,7 \\
Indice de gras & $\langle\mathrm{p} .100)$ & 2,2 \\
Rendement en muscle & $(\mathrm{p} .100)$ & 59,1 \\
\hline
\end{tabular}

Les carcasses étaient de bonne présentation, mais la couverture était un peu légère, comme on l'observe souvent sur les taurillons précoces.

A $219,3 \mathrm{~kg}$, elles se situent à $45 \mathrm{~kg}$ audessus de celles des Zébus Malgaches déjà cités, abattus à 19 mois environ, donc un peu plus âgés. On retrouve là une autre expression du gain obtenu.

Sur le plan économique cependant, la transformation de ces veaux métis de races laitières risque d'achopper. Comme nous l'avons déjà dit, ce sont les seuls veaux disponibles dans les villes des Hauts Plateaux. Leur prix est par conséquent élevé : de 100 FMG à 150 FMG le $\mathrm{kg}$ vif selon leur état général, ce qui amène à 15.000 FMG le prix d'un veau de $100 \mathrm{~kg}$ en état relativement correct.

Comme la commercialisation de viandes de type " baby beef " n'est pas courante à Madagascar, elle se trouve en concurrence avec le bœuf. L'avantage tiré de la plus grande tendreté générale, qui permet de préparer en cuisson rapide un beaucoup plus grand nombre de morceaux, demande pour être exploité l'assentiment des bouchers et des consommateurs. Cet assentissement n'a pas, évidemment, été obtenu dès le premier essai. Il n'est pas certain qu'il puisse l'être rapidement, compte tenu de ce qu'à Madagascar la clientèle européenne, consommatrice de viande grillée est peu nombreuse, et trouve satisfaction dans les morceaux nobles des bœufs âgés.

Il serait, dans le contexte actuel, sans doute plus logique d'essayer d'améliorer la qualité des veaux en tant que tels, c'est ce que font déjà certains professionnels de la viande.

\section{CONCLUSION}

Que ce soit par croisement avec un zébu amélioré tel que le Brahman, ou par croisement avec des taurins de races laitières on augmente considérablement le potentiel de croissance et on améliore les indices de consommation du zébu malgache.

La possibilité d'obtenir des carcasses suffisantes en un temps beaucoup plus court permettrait à l'engraisseur d'avoir, de surcrôt, des rotations plus rapides.

Le croisement Brahman, réalisable en extensif et donmant des veaux dans des conditions comparables au Zébu Malgache, paraît particulièrement recommandé.

\section{SUMMARY}

Possibilities of increase of malagazy zebu economical productivity by genetical improvment and intensive feeding

Brahman Zebu $\times$ Malagasy Zebu of about sixteen months cross-bred and Malagasy Zebu $X$ Friesland (or Norman) cross-bred animals of about six at seven months have been reared intensively.

Results of growth, feed efficiency and carcass characteristics are gived.

Comparatively to Malagasy Zebu of same age, fed in comparative way, important gains are obtained in all plans. 
Brahman crossing, realizable in extensive management, seems to be very recommanded.

\section{RESUMEN}

Posibilidades de aumento de la productividad económica del cebú malgacho por mejoración genética y alimentación intensiva

Se alimentaron intensivamente animales mestizos cebú Brahman $\chi$ cebú Malgacho de unos 16 meses de edad y mestizos cebú Malgacho $X$ Frison (o Normand) de unos 6 a 7 meses de edad. Se dan los resultados de crecimientos, indices de consumo, y las características de canales.

Comparativamente con cebues Malgachos de misma edad, alimentados de igual modo, se obtienen incrementos importantes.

El cruzamiento Brahman, posible en ganaderia extensiva, parece muy recomendado.

\section{BIBLIOGRAPHIE}

1. SERRES (H.) et Collab., "Essais d'engraissement de zébus à Madagascar», Rev. Elev. Méd. vét. Pays trop., 1971, 24 (3) : ...
2. SERRES (H.) et Collab., * Le croisement Brahman à Madagascar », Rev. Elev. Méd. vét. Pays trop., 1968, 21 (4): 519-61.

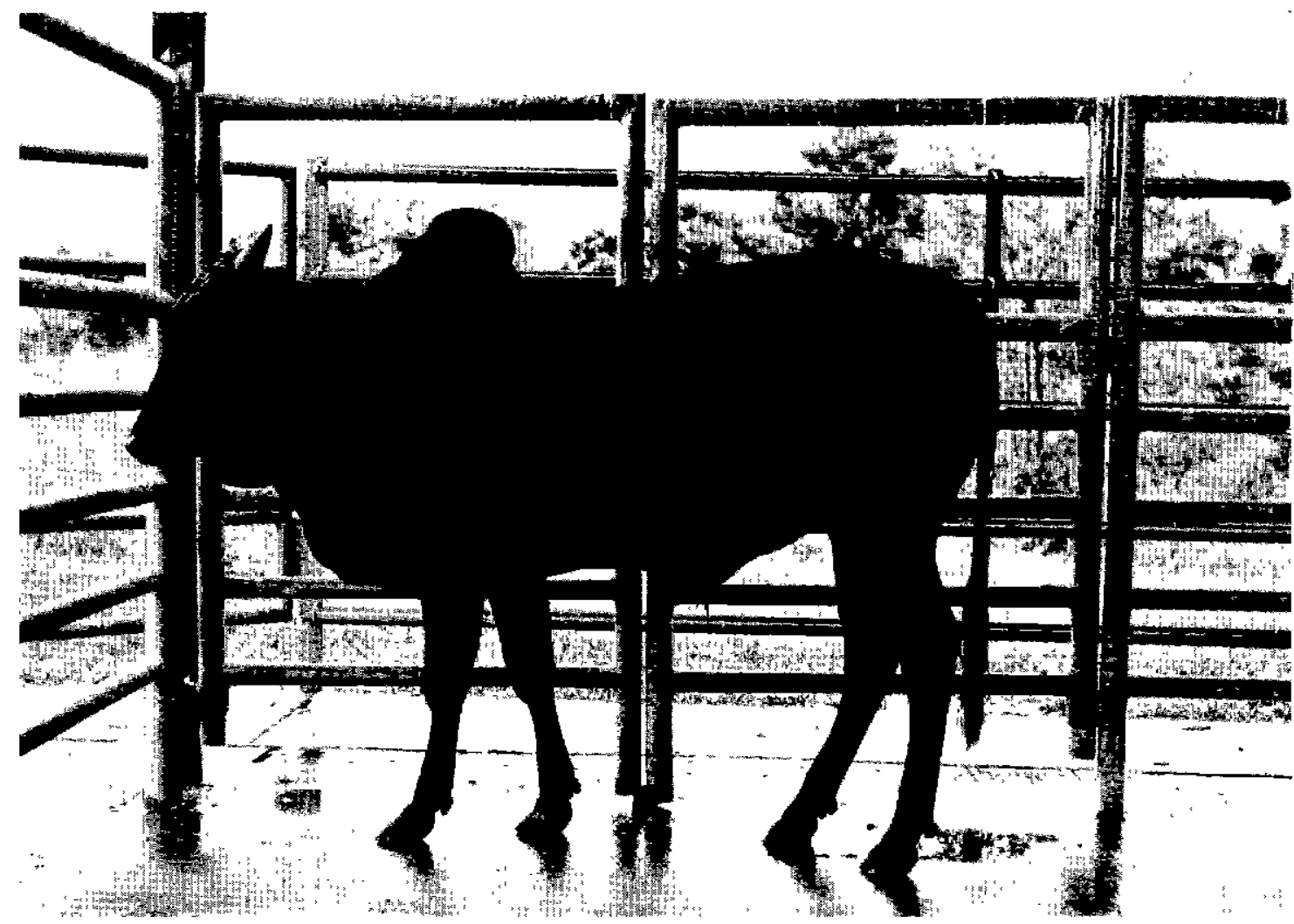

Photo 1. - Taurillon zébu malgache à 18 mois. 


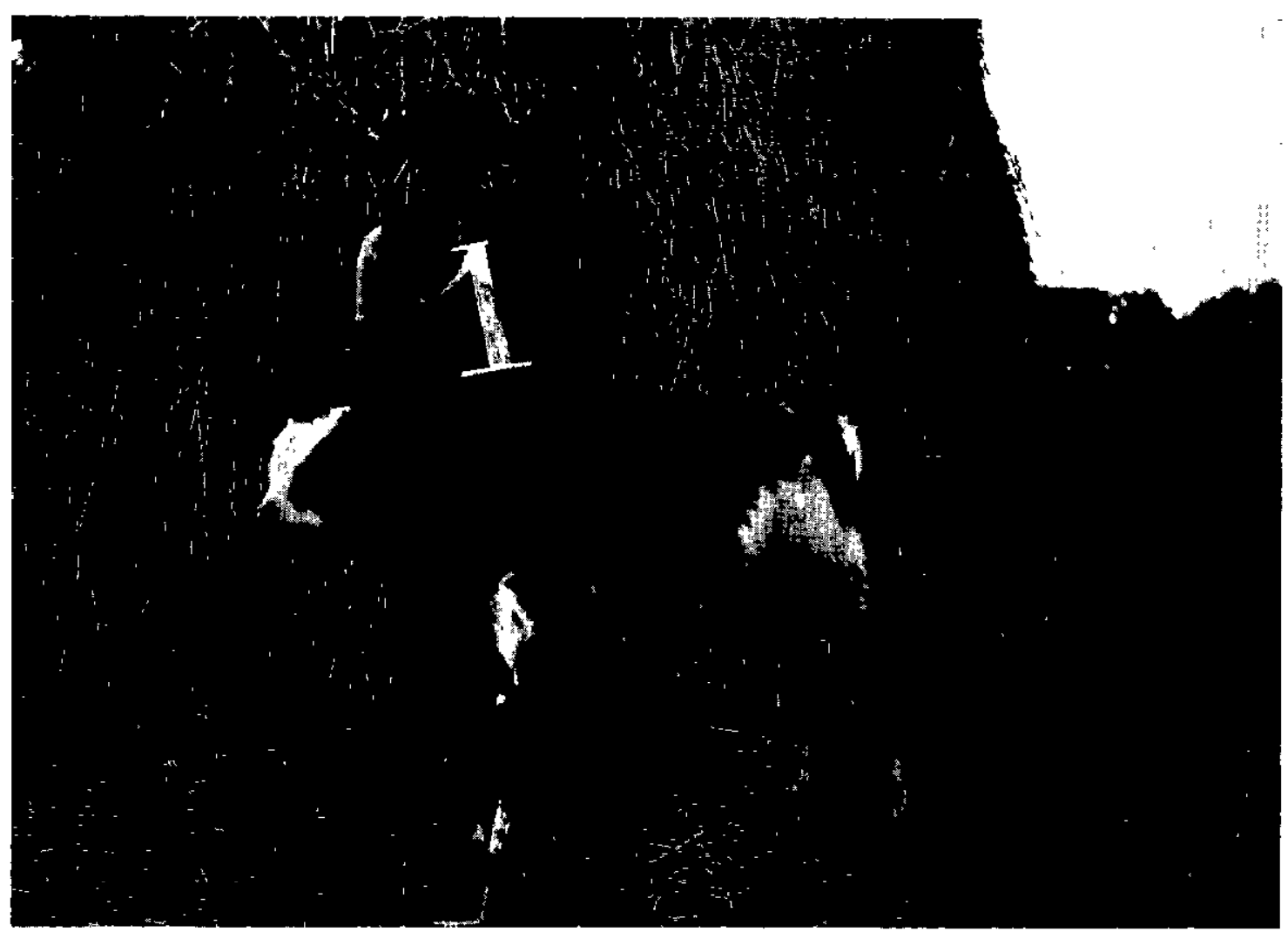

Photo 2. - Veau métis pie noir à 6 mois.

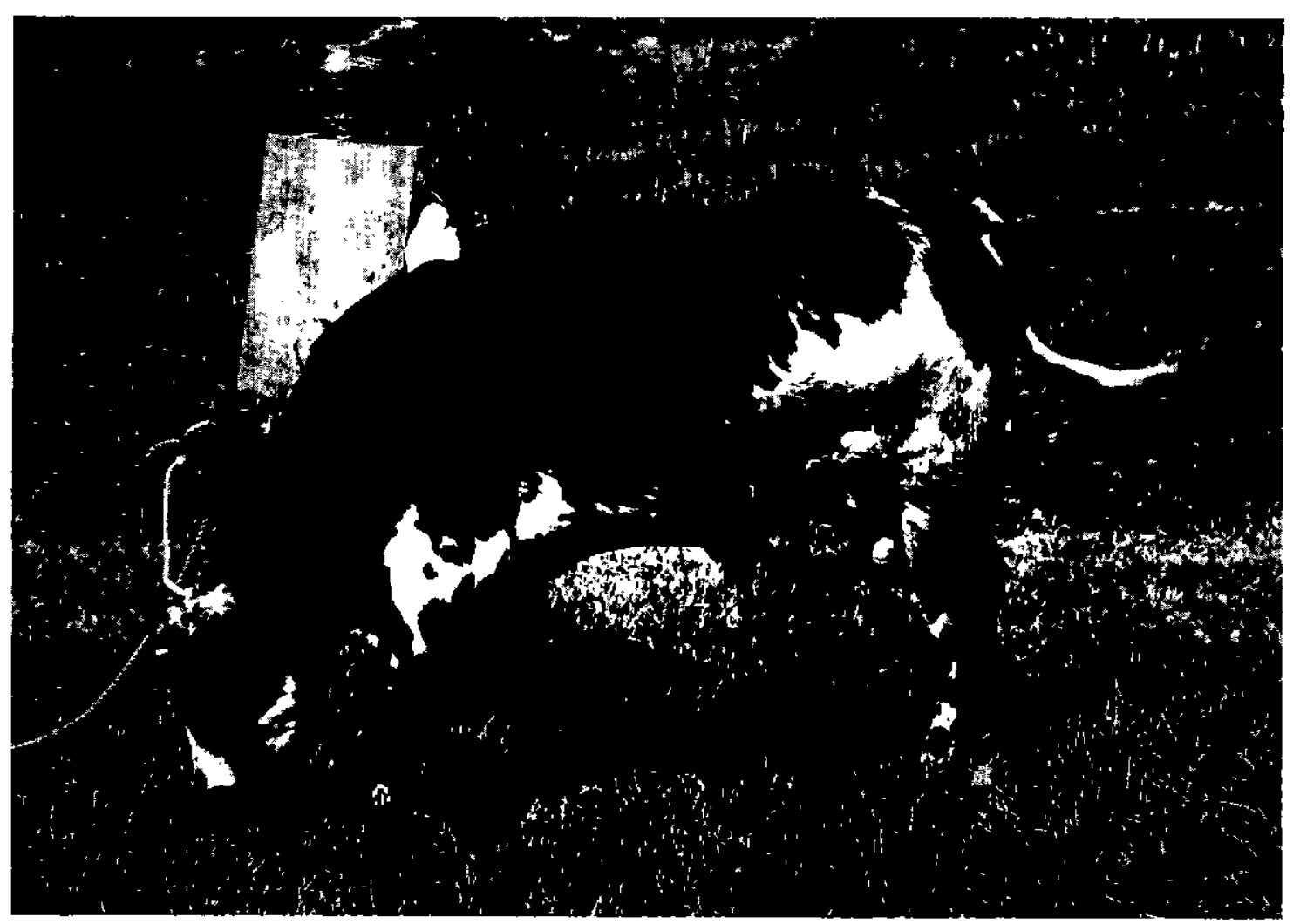

Photo 3. - Le même neuf mois plus tard. 


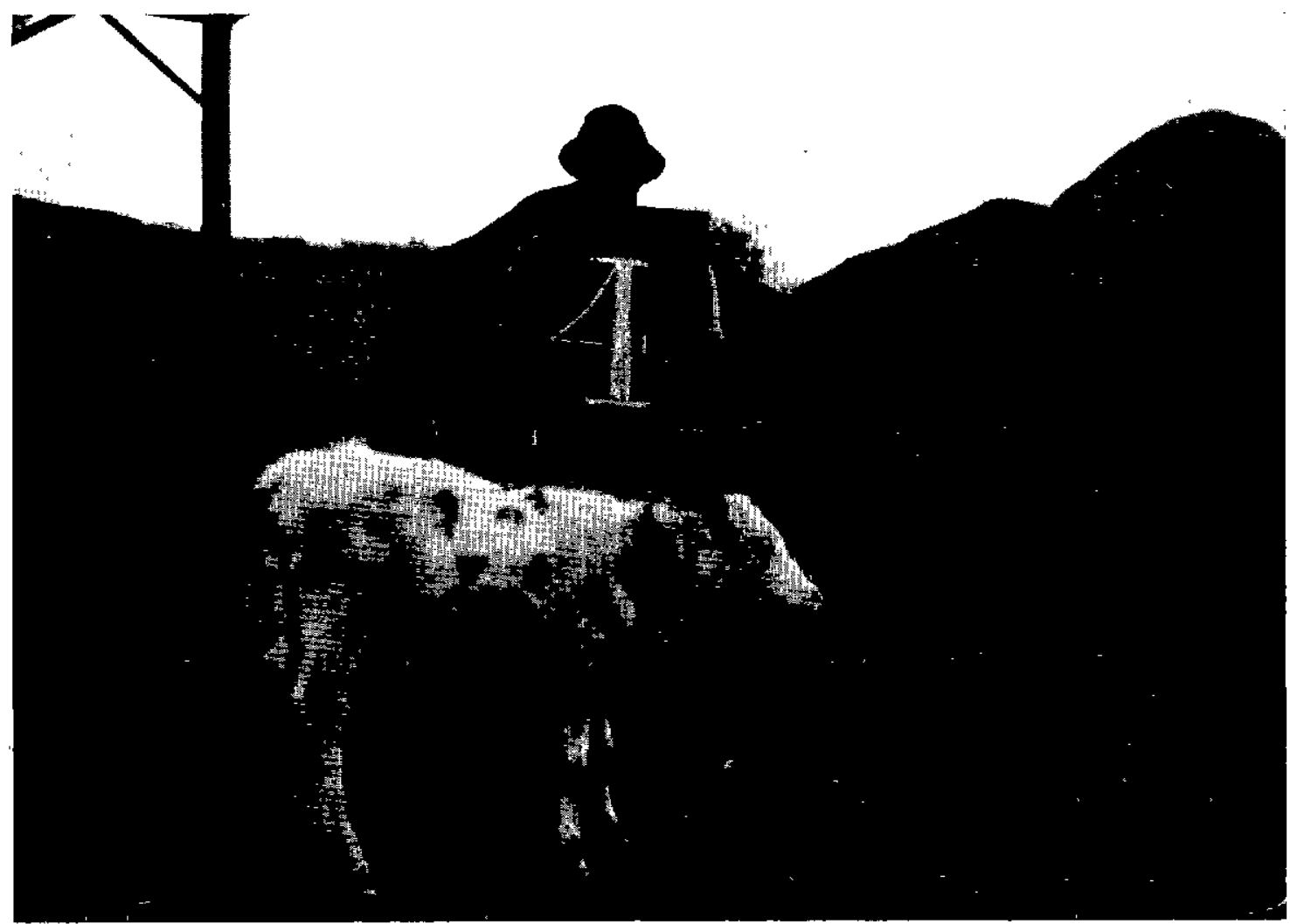

Photo 4. - Taurillon métis normand à 6 mois.

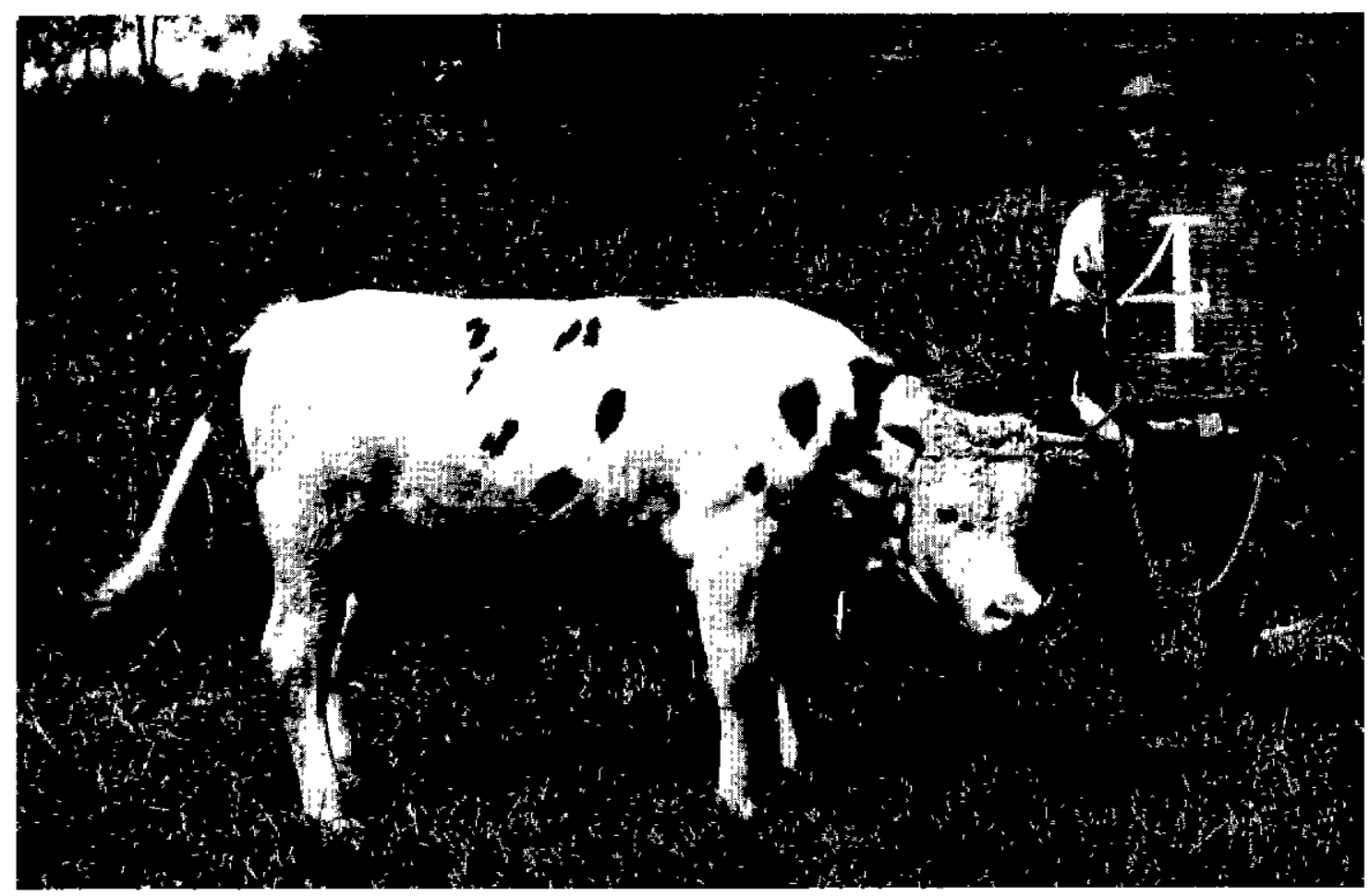

Photo 5. - Le même neuf mois plus tard. 